\title{
SMOOTH WINDS AND CLUMPED WINDS FOR
}

\section{EARLY-TYPE STARS}

\author{
R. BLOMME \\ Royal Observatory of Belgium, Ringlaan 3, B-1180 Brussels, Belgium \\ and \\ M. RUNACRES \\ Vrije Universiteit Brussel, Pleinlaan 2, B-1050 Brussels, Belgium
}

\begin{abstract}
The use of the IR and radio continuum as a clumping indicator for the stellar wind of early-type stars is investigated.
\end{abstract}

\section{Introduction}

The instability of hot star winds was already pointed out in 1970 (Lucy and Solomon) and has been confirmed in recent years by various observations. This instability causes the wind to be highly structured: it is clumped rather than homogeneous (Owocki et al. 1988). Free-free and bound-free scattering are the most important opacity sources at infrared and radio wavelengths and they depend on the square of the density. Hence the flux at these wavelengths can be used as a clumping indicator.

Furthermore, the infrared continuum is formed close to the surface of the star, whereas the radio continuum is formed far out in the wind. Hence by looking at different wavelengths, we can scan through different geometrical regions of the stellar wind. In this study we shall compare both the smooth models (density monotonously decreasing with radius) and clumped models ('wild' density variations) with observations.

\section{Results and Conclusions}

We applied our own NLTE model to a sample of stars (for details we refer to Runacres and Blomme 1994). We find good agreement between our model predictions and the observations of the few stars we already studied. As an example we show $\epsilon$ Ori (figure 1).

However, for the extreme $O$ star $\zeta$ Pup we find an infrared excess which is slightly too low (figure 2). This probably points to clumping due to instability in part of the stellar wind. To test this hypothesis we ran a model with a clumped density structure damping out before 10 stellar radii. It is clear that this produces an effect in the right direction: even though we have taken a rather arbitrary damping scale length, the clumped model agrees with the observations better than the smooth one does. 


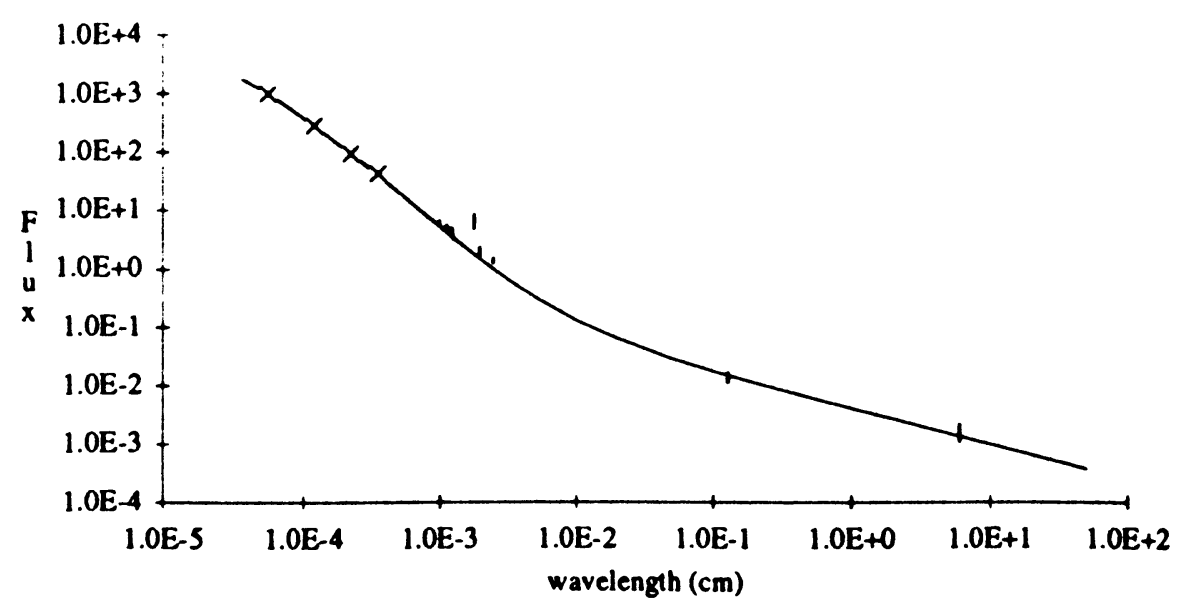

Fig. 1. Model fluxes (smooth line) for $\epsilon$ Ori compared to the observations

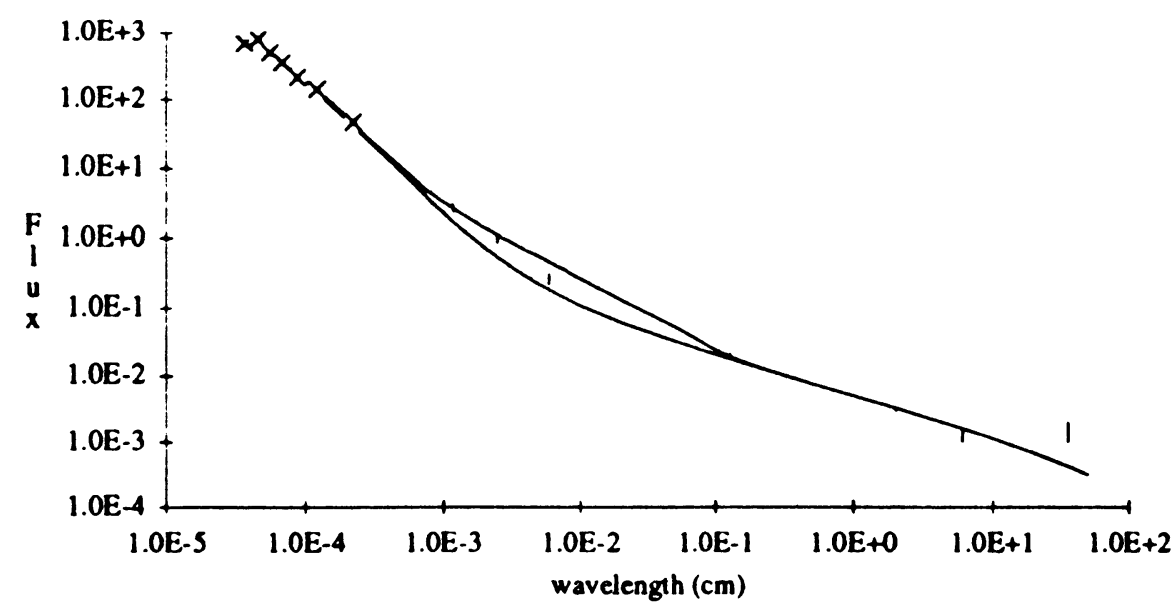

Fig. 2. Model fluxes for $\zeta$ Pup compared to the observations. The lower line shows the results for the smooth model, the upper one for the clumped model

It appears that clumping might not be all that severe or frequent among $O$ and $B$ stars. However, more definite conclusions will be drawn once the study is completed.

\section{References}

Lucy, L.B., Solomon, P.M.: 1970, ApJ 159, 879

Owocki, S.P., Castor, J.I., Rybicki, G.B.: 1988, $A p J$ 335, 914

Runacres, M.C., Blomme, R.: 1994, in Instability and Variability of Hot Star Winds, Kluwer, in press 